\title{
PARAMETRIC STUDIES OF THE RESPONSE DECREMENT PRODUCED BY MECHANICAL STIMULI IN THE PROTOZOAN, Stentor coeruleus
}

\author{
DAVID C. WOOD* \\ Brain Research Laboratory, The University of Michigan, Ann Arbor, Michigan 43104
}

\section{SUMMARY}

A decrement in the probability of eliciting a response is observed during the course of repetitive stimulation in many response systems of both metazoa and protozoa. Those forms of metazoan response decrement called habituation have recently been characterized behaviorally. In the studies reported here, the response decrement of the contractile protozoan, Stentor coeruleus, to repeated mechanical stimulation was systemically characterized to provide a comparison with metazoan habituation. This change in response probability was closely approximated by a negative exponential function after the first few stimuli. Animals responded to parametric variations of stimulus amplitude, interstimulus interval and retention period in a manner paralleling that observed in metazoa. However, neither interpolated large amplitude mechanical stimuli nor suprathreshold electrical stimuli produced dishabituation in Stentor though these stimuli are among the most effective dishabituating stimuli for metazoan tactile response systems. Behavioral analysis of the response decrement proceeded on the assumption that Stentor contains receptor and effector mechanisms only. Since the response decrement was not correlated with a change in responsiveness to electrical stimuli, the ability of the animal to contract appears to be unaltered by the process producing the response decrement. On the other hand, weak mechanical prestimuli were found to increase the rate of the response decrement which suggests that a process of receptor adaption is operative.

\section{INTRODUCTION}

In 1906, H. S. Jennings described the process of "acclimatization" in the protozoan, Stentor (Jennings, 1906). Stentor exists at rest in the form of a straight trumpet attached to the substratum at the narrow end

* Presently at the Department of Psychology, The University of Pittsburgh, Pittsburgh, Pennsylvania 15213. This work represents part of a dissertation submitted to the University of Michigan in partial fulfillment of the requirements for a Ph.D. degree. 
by pseudopodial outgrowths. Mechanical and electrical stimuli can produce an all-or-none contraction of the animal to a spherical form. Jennings observed that repeated mechanical stimuli of roughly constant amplitude became progressively less effective in producing this contraction. Subsequent writers (Harris, 1943; Thorpe, 1956) have noted the similarity of this behavioral modification to the phenomenon of habituation noted in metazoa. Only recently, however, have characterizations of metazoan habituation provided sufficient data to allow a more exacting comparison of the response decrement noted in Stentor and habituation. It is the object of these studies to generate data pertinent to such a comparison and to provide some analysis of the functional basis of this response decrement.

\section{METHODS}

Animals. Stentor coeruleus was supplied commercially. A single animal was isolated from this culture and a strain was derived from it. This strain was cultured at $20^{\circ} \mathrm{C}$ in an artificial medium containing: $2.5 \mathrm{mM} \mathrm{CaCl}, 1.0 \mathrm{mM} \mathrm{NaNO}_{3}, 1.0 \mathrm{mM}$ $\mathrm{MgSO}_{4}, 0.1 \mathrm{mM} \mathrm{KH} \mathrm{PO}_{4}$, and $0.7 \mathrm{mM}$ Tris $\mathrm{HCl}$ at $\mathrm{pH}$ 7.0 to 7.6. Rice grains served as nutrient for the cultures.

Experimental animals were chosen from randomly selected subcultures which were 7 to 11 days of age. In the 26 subcultures used the division rate averaged 1 division every 10 days over this 7 to 11 day period.

Apparatus and procedure. For behavioral testing, animals were placed in a $5 \mathrm{ml}$ beaker with $2 \mathrm{ml}$ of culture medium. The bottom of this test beaker was covered with ruled filter paper to which most of the animals became attached within several hours after being placed in the beaker. The beaker was fastened to the top of the armature of a vertically oriented solenoid with an adjustable calibrated displacement. Animals were mechanically stimulated by actuating the solenoid, which displaced the armature, and hence the beaker, rapidly downward. After $2 \mathrm{sec}$ the solenoid armature and test beaker were restored to their original position by a spring. Animals were observed through a stereomicroscope placed above the beaker.

About 15-20 animal were placed in the test beaker 8 to $20 \mathrm{hr}$ before an experimental session. After this time the position of individual sessile animals on the ruled filter paper was noted and the test series begun. When contracting in response to stimuli, animals generally remained attached to the filter paper, therefore it was possible to localize and record the behavior of individual animals throughout an entire testing session. Contractions of Stentor are easily discerned events involving a shortening of their body length by more than $50 \%$ in approximately 10 msec. (Wood, 1970). The probability of a contraction occurring spontaneously is very small.

Since the animals required 15 to $45 \mathrm{sec}$ to reextend, there was ample time to determine for a number of animals if they contracted to a particular stimulus. During the course of these experiments the average number of animals studied simultaneously during individual testing sessions was 14.8. However, during testing an average of 2.7 animals swam away from their point of attachment and their records had to be discarded as being incomplete.

For experiments requiring the use of electrical stimuli, a circular stainless steel electrode was placed at the bottom of the beaker and covered with ruled filter paper and a retaining ring. A stainless steel ring supported $1.0 \mathrm{~cm}$ above the bottom of the beaker served as the second electrode. Insulated silver leads from these electrodes were lead off to a calibrated stimulator. Using enlarged models of these electrodes, the electric field above the lower electrode was found to be uniform for a scale 
distance twice that occupied by the Stentor. Electrical stimuli were therefore assumed to have been uniformily applied to all animals on the bottom of the test beaker regardless of their exact position.

The apparatus was modified to permit both horizontal and vertical displacements of the test beaker to test for the "dishabituating" effect of a large amplitude mechanical stimulus. The test beaker was mounted directly above a vertically oriented solenoid at the end of a $12.5 \mathrm{~cm}$ long flexible metal strip whose other end was fastened to the solenoid armature. A second flexible strip of the same length as the first and at a right angle to it connected the mounting at the base of the beaker to the armature of a horizontally oriented solenoid. Actuation of the vertically oriented solenoid moved the beaker vertically a calibrated distance, while actuation of the horizontally mounted solenoid moved it in a horizontal direction.

\section{RESULTS}

General behavior pattern. During the course of the following experiments one experimental procedure was applied 31 times and data on the behavior of 414 animals exposed to this procedure was collected. In this procedure the displacement of the solenoid was set for $0.10 \mathrm{in}$. and the solenoid was actuated once every minute for one hour. In the following section data for animals exposed to this stimulation procedure is taken from the experiments to be reported subsequently and analyzed collectively. From these data certain general statements can be made about the contractile behavior of Stentor in response to repeated mechanical stimulation.

No animal responded to all the stimuli or failed to respond to at least one. $401(96.9 \%)$ responded more frequently to the first ten stimuli than to the last ten stimuli. Thus, a response decrement was nearly universal among the animals tested.

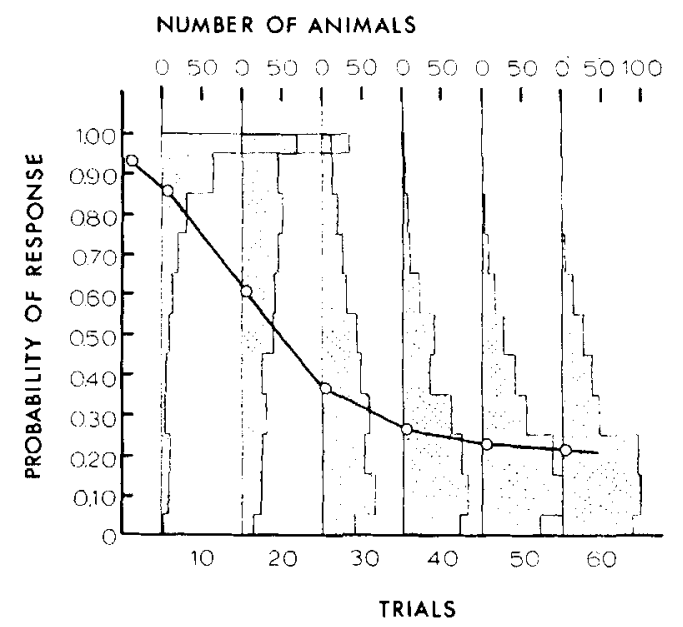

Fig. 1. Average probability af response and variability of response to 60 mechanical stimuli of amplitude $0.10 \mathrm{in}$. and frequency $1 / \mathrm{min}$. In this and subsequent graphs the first point represents the probability of response to the first stimulus and subsequent points represent the average probability of response to 10 stimuli. 
Figure 1 graphically presents changes in average response probability and variability in response observed among the 414 animals during the hour of testing. After the first 10 stimuli the curve follows an exponentially falling course until an asymptote near 0.20 is approached (Figure 2). Response probability does not appear to approach a zero level of response, rather an asymptote does appear to exist.

Sources of the variance between animals evident in Figure 1 during the last 20 trials were studied by means of one-way analyses of variance. Only variance correlated with the subculture medium from which the animals came contributed significantly to the total variance (accounting for $20 \%$ of it). Variables such as position of the animals in the test beaker, age of the subculture on the day of testing, and subculture division rate did not account for a significant amount of the variance.

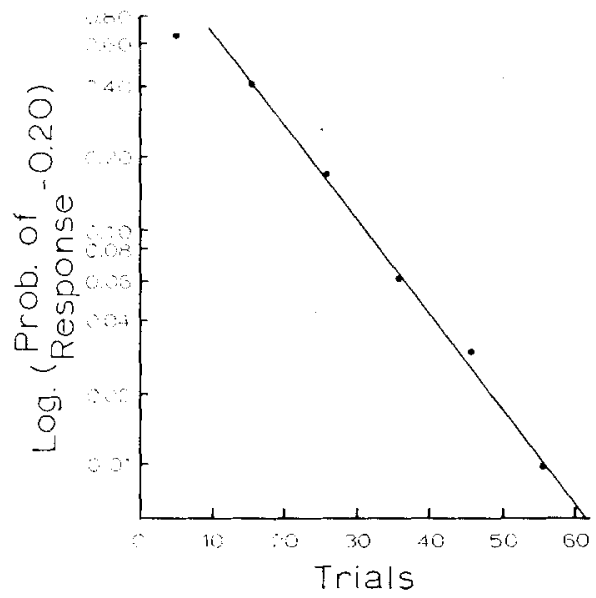

Fig. 2. Logarithmic plot of the probability of response less the asymptotic probability of response showing that the observed response decrement curve is approximated by a negative exponential function of the number of trials.

412 of the 414 animals tested responded one or more times following their first non-response, thus the response probability of individual animals did not change from 1.0 to 0.0 in a step-wise fashion. After their first non-response animals continued to show a decrease in probability of response as the trials continued. Therefore, the decrease in probability of response appears to be a decremental process.

Parametric analysis of the response decrement. Numerous studies concur in demonstrating that the rate of response decrement and asymptotic response level is a function of the intensity or amplitude of the stimulus reiteratively employed. Weak or small stimuli are productive of more rapid and pronounced decrements than are strong or large stimuli. The effect of parametric variation of stimulus amplitude upon the pattern of response decrement was explored by exposing different 
groups of animals obtained from the same subcultures to one hour of $1 /$ min stimulation with amplitude settings of $0.10,0.06,0.04$, and 0.02 in.

The results are graphically displayed in Figure 3 . All points for a given block of 10 trials are significantly different at the 0.01 level or better (sign test) except those points on the 0.10 and 0.06 in. curves after 20 min of stimulation. Only a small and relatively constant number of animals swam in the various groups, hence these animals could contribute but little to the observed differences between groups. Further, data taken from the records of animals which later swam indicated that their

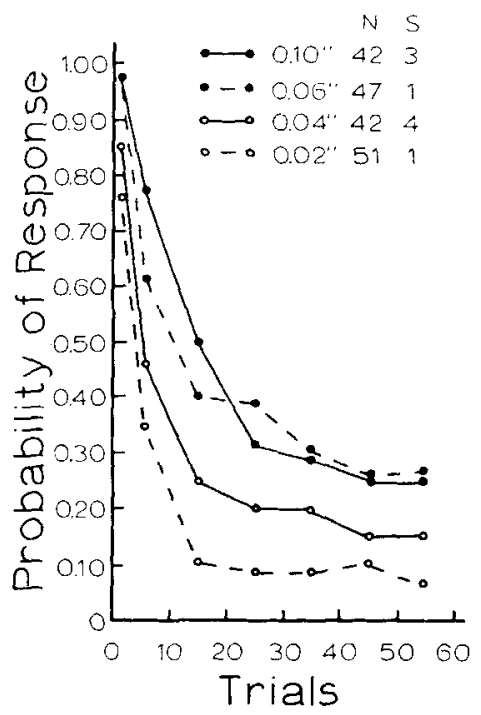

Fig. 3. Comparison of response decrement rates and asymptotes for mechanical stimuli of $0.10,0.06,0.04$, and 0.02 in. The number of animals comprising each group is given under the " $\mathrm{N}$ " and after the symbol designating the significance of each line. This is followed by a number under the " $S$ " indicating how many of the original animals swam before testing was complete. 'Thus, 45 animals were initially tested at $0.10 \mathrm{in}$.; 3 swam during testing, and 42 remained for the entire 60 trials.

probability of response was not significantly different from that of the remaining animals. It may be concluded that small amplitude stimuli produce more rapid and pronounced decrements than large amplitude stimuli.

As in the case of stimulus amplitude, a parametric study of interstimulus interval was conducted to ascertain if the behavior pattern of Stentor conforms to the behavior pattern exhibited by other animals. Animals were stimulated using interstimulus intervals of 1,2, and $3 \mathrm{~min}$ for 60 stimuli of amplitude 0.10 in. Shorter interstimulus intervals could not be employed because the animals frequently required 30 to $45 \mathrm{sec}$ to reextend and reextension was prerequisite to reliable observation of stimulus-induced contractions. 


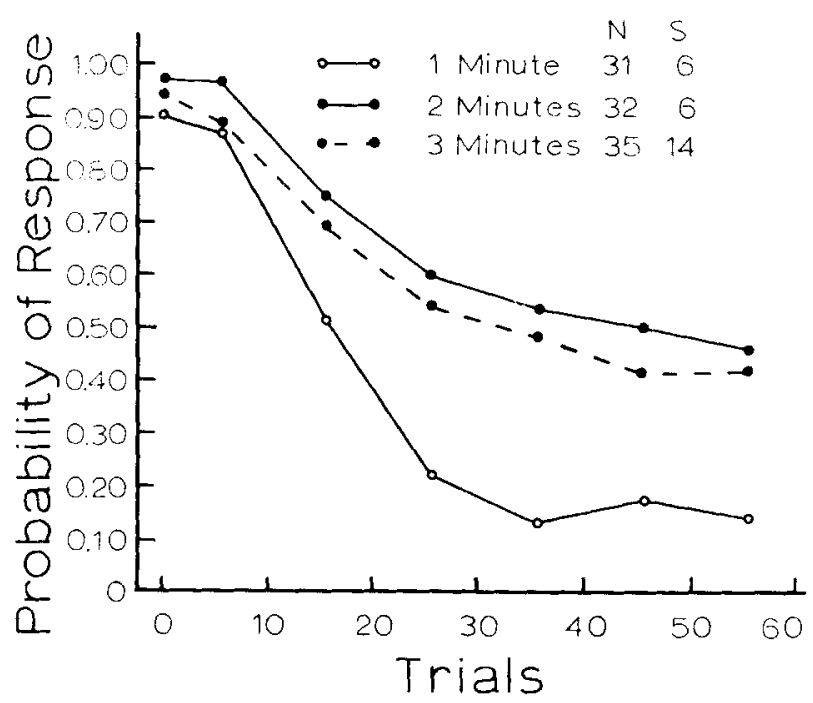

Fig. 4. Comparison of response decrements occurring to $0.10 \mathrm{in}$. stimuli presented at interstimulus intervals of $1 \mathrm{~min}, 2 \mathrm{~min}$, and $3 \mathrm{~min}$. Designation of number of animals in the various experimental groups and number of animals which swam away is as indicated in Figure 3.

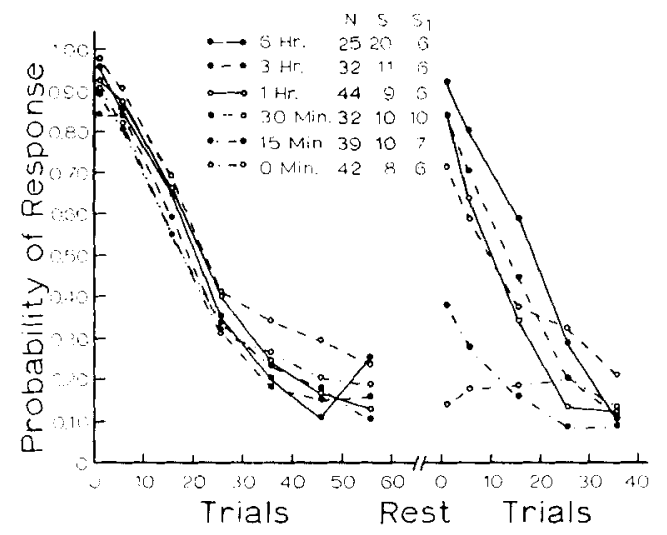

Fig. 5. Response decrements and subsequent recovery of responsiveness following $0-, 1,-, 1 / 2-, 1-, 3-$, and $6-\mathrm{hr}$ rest periods. The designation of number of animals which were tested and which swam is as previously described. In addition the number of animals which swam during the First 60 trials is given under " $\mathrm{S}_{1}$."

The response decrement was more rapid and pronounced for stimuli delivered with an interstimulus interval of $1 \mathrm{~min}$ than for stimuli delivered with interstimulus intervals of 2 or 3 min as shown in Figure 4. Experimental points on the 2 and 3 min curves do not differ significantly from one another (sign test).

Habituated responses show spontaneous recovery during periods in which no stimuli are applied. The relationship between response prob- 


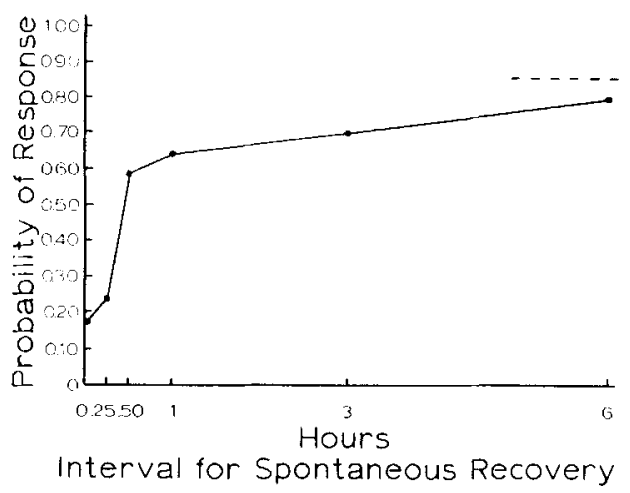

Fig. 6. Probability of response on the first $\mathbf{1 0}$ retest trials as a function of the interval for spontaneous recovery rest interval. Dashed line indicates the average probability of response on trials $1-10$ of the initial stimulation series.

ability or amplitude, and the duration of the rest period is characteristically a negatively accelerated monotonic function. In order to determine the pattern of spontaneous recovery of Stentor, 18 groups of animals were subjected to 60 mechanical stimuli of amplitude $0.10 \mathrm{in}$. occurring at a frequency of $1 / \mathrm{min}$. Three randomly assigned groups were retested with a series of $400.10 \mathrm{in.} 1 / \mathrm{min}$ stimuli after each of 6 stimulusfree intervals: $0,1 / 4,1 / 2,1,3$, and $6 \mathrm{hrs}$. Stimulus-free periods of greater length were not attempted because a large percentage of the animals altered their position in the test beaker during the course of the $6 \mathrm{hr}$ rest period. Only data from animals which remained sessile on the ruled filter paper throughout the entire period of testing was used in the following analyses.

Animals mechanically stimulated after the several rest periods showed decreased probabilities of response to the second series of stimuli (Figure 5). The probability of response to the first 10 stimuli of the retest session is plotted as a function of the rest interval in Figure 6. Statistical analysis of the data incorporated into this latter figure shows there was a significant reduction of initial response probability on retest as compared to the first series of stimuli for all rest periods shorter than and including $3 \mathrm{hrs}$ ( $\mathrm{p}<0.01$, sign test). Initial response probability for the 6 -hr retention group was not significantly lower on retest than during the first trials. The form of the curve described in Figure 6 is characteristic of spontaneous recovery from habituation.

While the 6-hr retention group did not show a significant depression of initial response probability, it did reach a 7 non-response out of 10 trials criterion with significantly fewer stimuli during retention trials than during the initial training trials ( $p<0.005$; $t$ test). For all groups the response decrement was more rapid during the second series of stimuli.

It has been claimed that dishabituation produced by intense stimuli is as ubiquitous a phenomenon as habituation itself (Thompson and 


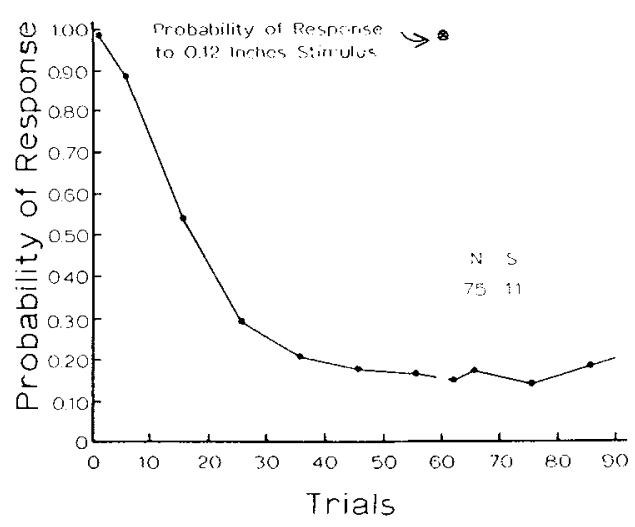

Fig. 7. Effect of a 0.12 in. mechanical stimulus on the response decrement produced by 0.04 in. mechanical stimuli. The designation of numbers of animals which were tested and which swam is as previously described.

Spencer, 1966). To test for dishabituation Stentor were initially exposed to $600.04 \mathrm{in.} 1 / \mathrm{min}$ stimuli to produce a response decrement. A larger amplitude "dishabituating" stimulus was used on trial 61 only. This stimulus was a 0.12 in. displacement of the beaker in a horizontal direction in contrast to the vertical displacements previously employed. The horizontal displacement was employed to avoid resetting the solenoid which procedure had previously been found to be unsatisfactory. Horizontal and vertical displacements of the test beaker were achieved by modifying the apparatus as described previously. One minute after the large amplitude stimulus the first of a train of $290.04 \mathrm{in.} 1 / \mathrm{min}$ vertically oriented displacements was applied. These stimuli were of the same amplitude and repetition rate as those previously employed.

The results are plotted in Figure 7. The responsiveness of these animals to the 0.04 in. stimuli was markedly greater than the responsiveness of the groups of animals used previously, probably owing to increased vibration in the modified apparatus. No evidence for dishabituation was obtained on trial 62 or subsequent trials. It might be argued that the excitatory effect of the large stimulus had dissipated during the minute following its application; even with this qualification it is evident that a dishabituation phenomenon of the sort tested must be very weak or nonexistent in Stentor.

Animals subjected to a series of 60 horizontal displacements of the beaker showed the same low probability of response to the first of a series of vertical displacements of the same amplitude as they did to the final 10 horizontal displacements. Conversely, response decrements produced by vertical displacements were also still apparent when horizontal displacements were employed subsequently. These results imply that horizontal and vertical displacements of the beaker produced similar effects on the animals. Hence, it presently seems unlikely that a large 
amplitude vertical displacement of the test beaker would have produced a greater degree of dishabituation than that produced by the horizontal displacement. A suprathreshold electrical stimulus also failed to produce dishabituation.

Many experimenters have demonstrated that habituation to a stimulus of one modality does not alter the animal's sensitivity and rate of habituation to subsequent stimuli in other modalities. Stentor and other contractile protozoa exhibit a contraction threshold when stimulated by electrical pulses. This circumstance allows one to test if the response decrement produced by mechanical stimuli interacts with this electrical threshold.

The experimental session began with a single descending series of 0.1 msec monophasic square pulses delivered at a rate of $1 / \mathrm{min}$ beginning with a $28 \mathrm{~V}$ pulse and ending with a $4 \mathrm{~V}$ pulse with $2 \mathrm{~V}$ steps spaced between pulses. The electrical stimuli were presented in descending order so that the threshold determination occurring after the mechanical stimulation would begin with the large amplitude electrical stimuli, hence an elevation of threshold would be immediately apparent. After the first threshold test a $15 \mathrm{~min}$ pause was allowed before $600.10 \mathrm{in} 1 / \mathrm{min}$ mechanical stimuli were presented. Immediately after these mechanical stimuli a second descending series of electrical pulses was used.

The 21 animals exhibited a typical curve of response decrement responding to 9.6 of the first 10 mechanical stimuli and only 3.4 of the last 10 mechanical stimuli. Their electrical threshold before the mechanical stimuli was $13.1 \mathrm{~V}$ as calculated by the Spearman method (Woodworth, 1936) and their final threshold was $13.8 \mathrm{~V}$. These thresholds are not significantly different $(t=0.30 ; t$ test). Thus, the repetitive mechanical stimuli did not appear to alter the electrical threshold of the animals measured in this fashion.

The first electrical stimulus presented after the mechanical stimuli had a voltage which was minimally sufficient to cause all the animals to contract during the initial threshold test. The first stimulus of the second threshold test also caused all the animals to contract. The second stimulus when employed in the initial threshold test produced contractions in 18 of the 21 test animals; after the mechanical stimuli 18 of the 21 animals again contracted to this stimulus. 17 animals contracted to the third stimulus initially, while after the mechanical stimuli 18 responded. The similarity in sensitivity of the animals to the first several electrical stimuli is particularly important since the response to these stimuli demonstrated the animals' condition immediately after the mechanical stimuli when their condition was not influenced by possible effects of the electrical stimuli and the passage of time involved in their presentation.

Stimuli, which do not themselves produce responses, can reduce an animal's probability of response to subsequent stimuli. Two experimental paradigms have been employed to demonstrate this phenomenon. Employing the most straight-forward of these paradigms, R. B. Clark 
(1960) showed that subthreshold prestimulation of Nereis increased the animals' rate of habituation to subsequent stimuli in the same modality. This phenomenon was called "latent" habituation. The second paradigm is that of "below-zero" habituation in which less spontaneous recovery is observed after stimulation in excess of the amount needed to produce a zero level of responding (Humphrey, 1933; Thompson and Spencer, 1966).

In the following experiment "latent" habituation was attempted by initially exposing the experimental animals to $300.004 \mathrm{in.} 1 / \mathrm{min}$ stimuli. 0.004 in. represents the smallest calibrated displacement possible with this apparatus, but some animals nevertheless responded to this stimulus. Control animals received only the $600.10 \mathrm{in.} 1 / \mathrm{min}$ stimuli.

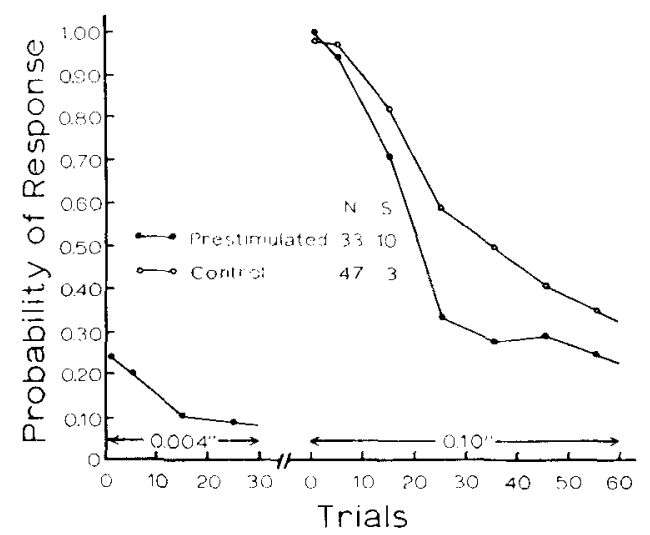

Fig. 8. Comparison of the response decrement produced by $300.004 \mathrm{in} .1 / \mathrm{min}$ stimuli followed by $600.10 \mathrm{in.} 1 / \mathrm{min}$ stimuli and that produced by $600.10 \mathrm{in} .1 \mathrm{~min}$ stimuli alone. Designation of numbers of animals which were tested and which swam is as previously described.

The prestimulated animals showed a decrement in responding to the 0.10 in. stimuli which was more rapid than that exhibited by the controls (Figure 8). This difference in responsiveness does not appear to be due to the differential loss of animals since the animals which swam in the prestimulated group were slightly less responsive to the mechanical stimuli than were the animals which remained. However, animals from these subcultures were generally more responsive to the mechanical stimuli than groups previously tested.

While the difference in response decrements between the two groups is apparent in Figure 8, the source of this difference is not apparent. It can be argued that the sum of effects from contractions to the prestimuli and testing stimuli determined the animals' final response probability. However, the prestimulated animals produced significantly fewer contractions to 90 stimuli than did the control animals to 60 stimuli i $p<$ 0.02 , t test). Thus, the prestimulation resulted in a greater response 


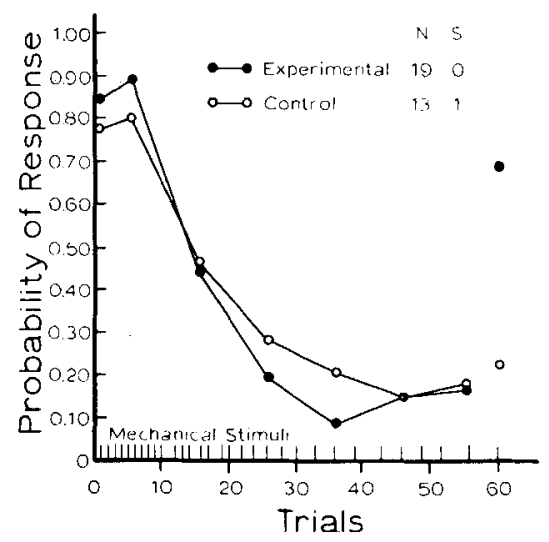

Fig. 9. Comparison of response patterns of animals stimulated with the pattern of mechanical stimuli shown along the abscissa (experimental group) and animals given $60 \mathrm{1} / \mathrm{min}$ stimuli of the same amplitude (control group). Designation of numbers of animals which were tested and which swam is as previously described.

decrement with fewer contractions than did the stimulation procedure normally applied.

The following experiment was designed to ascertain whether the number and sequence of contractions or number of stimuli was the more important determinant of the response decrement. In this experiment mechanical stimuli were presented to the experimental animals in such fashion that the resultant curve of response probability against time approximated that of the control animals stimulated once every minute. To accomplish this experimental animals were stimulated with 310.10 in. mechanical stimuli in the temporal sequence illustrated at the bottom of Figure 9.

The curve of response probability for the experimental animals fell below that of the control animals in some places (Figure 9). Likewise, during the first through the 59th $\mathrm{min}$ the number of contractions produced by the experimental animals (18.7) was fewer than the number of contractions produced by the controls (20.5). This disparity between experimental and control groups is attributable to 2 experimental animals; one of which only contracted twice while the other contracted 4 times. There were no animals with similar low frequencies of response in the control group. Even including these animals there is no significant difference between the groups in number of contractions per animal $(t=0.90 ; t$ test). Further, probabilities of response of the two groups during min 41 to 59 are very similar. Therefore the two procedures produced numbers of contractions and curves of response probability versus time which were rather well equated.

13 of 19 experimental animals $(69 \%)$ contracted to the stimulus presented at the 60th min; 3 of 13 control animals (23\%) contracted to this stimulus. This difference is significant at the 0.05 level (Fisher's exact 
probability test). Thus, differences in probability of response can be produced by varying the pattern of stimulation while keeping the number and sequence of contractions roughly constant.

\section{DISCUSSION}

The preceding parametric characterization of the response decrement observed in Stentor provides a basis for the comparison of this process with the phenomenon of habituation observed in metazoa. If a strong similarity exists between protozoan and metazoan behavioral modifications then it is reasonable to expect that similar physiological and biochemical bases may underlie these behavioral changes.

The comparison of metazoan and protozoan behaviors is greatly facilitated by the fact that Thompson and Spencer (1966) have recently formulated a parametric characterization of metazoan habituation which serves as an operational definition of it. It appears adequate for the purposes of comparison to enumerate the reported experimental results from Stentor and to note their degree of similarity or dissimilarity with the appropriate proposition of Thompson and Spencer as follows:

(1) The curve of response probability versus trials for Stentor shows a clear and pronounced decrement in response probability with repeated application of a mechanical stimulus. This curve is well-fitted by a negative exponential function after the first 10 trials.

(2) Following 600.10 in $1 / \mathrm{min}$ stimuli, Stentor showed a negatively accelerated curve of spontaneous recovery. Responsiveness was almost completely recovered after $6 \mathrm{hrs}$.

(3) The response decrement occurred more rapidly to stimuli applied after the period allowed for spontaneous recovery.

(4) As the interstimulus interval of the mechanical stimuli was raised from $1 \mathrm{~min}$ to $2 \mathrm{~min}$, the rate of the response decrement decreased and the asymptotic probability of response increased.

(5) The probability of response to stimuli of large amplitude was initially greater, decreased more slowly, and attained a higher asymptotic level than the probability of response to small amplitude stimuli.

The preceding five qualitative statements agree with the first five parametric relations which Thompson and Spencer (1966) feel characterize habituation. Further, the length of the interstimulus interval and the time period required for spontaneous recovery appear to be in quantitative agreement with similar values in metazoan studies.

While qualitatively the behavior of Stentor and that of other contractile protozoa is similar, nevertheless quantitative differences are apparent. For example, Vorticella and Spirostomum reextend after a contraction in but a couple of seconds, while Stentor requires 15 to $45 \mathrm{sec}$ to reextend. After a series of mechanical stimuli, Spirostomum shows more or less complete spontaneous recovery in only $30 \mathrm{~min}$ (Kinastowski, 1963b). Finally, both Spirostomum and Vorticella will show response decrements 
to mechanical stimuli only if these are presented with interstimulus intervals less than 1 min (Kinastowski, 1963a; Danisch, 1921). It therefore appears that the processes governing reextension and recovery of responsiveness operate more rapidly in Spirostomum and Vorticella than in Stentor.

(6) A "latent" response decrement was produced by small amplitude mechanical stimuli applied before the regular test sequence. As noted previously the "latent" response decrement appears related to the phenomenon of "below-zero" habituation. Thompson and Spencer's parametric relation Number 6 specifies that habituation "may proceed beyond the zero or asymptotic level." This possibility was not directly explored; however, since a "latent" response decrement was observed and since Kinastowski (1963b) observed a "below-zero" effect in Spirostomum, it seems probable that such a phenomenon would be found in Stentor.

(7) The response decrement produced by 60 mechanical stimuli did not alter the animal's response probability to electrical test stimuli. Most authors (eg., Thorpe, 1956; McConnell, 1966) in defining habituation have considered that stimulus specificity of the kind observed in Stentor is an essential aspect. It is therefore somewhat surprising that Thompson and Spencer specify that some degree of stimulus generalization be observed in response decrements called habituation. Stimulus generalization from a habituating stimulus to a test stimulus has been shown in metazoa, with one exception, only for stimuli presented in the same modality. A response decrement produced by using a horizontal displacement of the test beaker as a stimulus generalized to stimuli produced by moving the beaker in a vertical direction and conversely. However, it seems likely that these stimuli were so coarse as to stimulate all the receptor surfaces of the animals which resulted in the observed interaction between the response decrements. Applying the "habituating" stimulus to one constrained surface area of a single Stentor and testing for interaction with a similar test stimulus applied to a different surface area might be thought to circumvent this problem. In this case, however, the contractions produced by the "habituating" stimulus might act to produce a partial response decrement to the test stimulus, since the contractions produce movement, distortion, and hence mechanical stimulation of the animals' entire surface area. In view of this possibility a simple experiment testing interaction between two response decrements produced by different mechanical stimuli did not appear possible.

(8) No increase in responsiveness to small amplitude stimuli was observed after a large mechanical stimulus had caused the animals to contract. Similarly, dishabituation was not produced by a suprathreshold electrical stimulus. Since these two forms of producing dishabituation are among the most effective means of producing dishabituation in metazoa, it appears probable that dishabituation cannot be produced in Stentor. The correspondence of Stentor's behavior to Thompson and 
Spencer's parametric relations Numbers 8 and 9 is dependent on the observation of such a dishabituation phenomen. The failure to observe dishabituation in Stentor does not exclude its response decrement from the category of processes called habituation, since Thompson and Spencer note in their article "Parametric relations Numbers 8 and 9 are not really relevant to habituation as such, but rather describe a separate process." It therefore appears reasonable to conclude that Stentor may possess a rudimentary habituation mechanism without an additional mechanism for sensitization and hence dishabituation.

In sum, the behavioral characteristics of the response decrement observed in Stentor appear to be congruent with the Parametric relations accorded the simple habituation process by Thompson and Spencer.

Aside from providing a characterization of the response decrement, some of the experimental results are suggestive of its basis. To make this type of analysis it is necessary to characterize the processes which appear to be operative within the animal between the time of stimulation and response. It has previously been noted that Stentor contracts in an all-or-none fashion to mechanical and electrical stimuli (see alro Wood, 1970). However, animals do not contract to all stimuli, hence contraction amplitude is not a direct function of stimulus amplitude or presence. To explain this lack of correlation between stimulus and response, it seems necessary to postulate some internal mechanism(s) which generates the contractions in an all-or-none fashion and which is either actuated or not actuated by the stimulus. This hypothetical process(es) is here referred to as the effector process.

Stimulus energy must actuate the effector process since contractions occur immediately aiter stimuli. Since the stimulus energy is mechanical and the contractions apparently involve some biochemical reactions, there must be some transduction involved. This transduction process(es) is hereafter referred to as the receptor or sensory process.

The response decrement observed does not appear to be entirely or even largely due to fatigue of the effector process. In the final experiment it was observed that the number of contractions during one hour could be held constant while the level of the response decrement varied. Thus, the response decrement is not a function of the number of contractions produced as an effector fatigue hypothesis would suggest. Secondly, effector fatigue should decrease the animal's ability to contract to all modalities of stimuli. If the response decrement produced by mechanical stimuli is produced by an increase of effector fatigue, then the ability of the animals to contract to electrical stimuli should be depressed. This result was counter-indicated by the experiment in which electrical thresholds were measured.

If effector fatigue does not provide an adequate explanation of the entire response decrement, then it must be supposed that some alteration of the receptor process is involved. In accord with this conclusion, prestimulation was found to reduce the number of contractions required to 
produce the response decrement. Similarly, the final experiment showed that the response decrement was at least in part a function of the number of stimuli received rather than of the number or sequence of contractions produced. Therefore, activation of the hypothetical receptor process by the mechanical stimuli must have been instrumental in producing much of the response decrement observed. This result is explicable on the assumption that a decrement in sensory or receptor function is the principle basis of the response decrement.

Definitions of habituation specifically exclude receptor adaptation or effector fatigue as the basis of the decrement. In this regard the phenomenon observed in Stentor appears to differ from that in metazoa. However, it appears probable that the response decrement in Stentor does not satisfy this aspect of the definition of habituation simply because Stentor is unicellular. The behavioral properties of Stentor should perhaps be more appropriately compared to those of single cells, specifically neurons, in metazoa. Thompson and Spencer (1966), Spencer et al. (1966, a, b) and Buchwald et al. (1965) have found that single neurons display response characteristics to repeated stimulation which mirror the process of habituation observed in the intact animal. Sharpless (1964) has suggested that habituation observed at the neuronal level may be due to post-synaptic membrane desensitization. Since the postsynaptic membrane is in effect a chemoreceptor, this model suggests that habiuation involves receptor desensitization at the level of the single cell. Thus, receptor adaptation is presently a satisfactory model for habituation provided the receptors are surface areas of central neurons. This being the case it would appear that the response decrement observed in Stentor may have a common functional basis with metazoan habituation, being distinguished from it only because the desensitized receptor surface does not belong to a central neuron.

\section{REFERENCE}

Buchwald, J. S., Halas, E. S., and Schram, S. (1965). Progressive changes in efferent unit responses to repeated cutaneous stimulation in spinal cats. $J$. Neurophysiol. 28: 200-215.

Clark, R. B. (1960). Habituation of the polychaete Nereis to sudden stimuli. 1. General properties of the habituation process. Anim. Behav. 8: 82-91.

Danisch, F. (1921), Über Reizbiologie und Reizempfindlichkeit von Vorticella nebullifera. Z. allg. Physiol. 19: 133-190.

HaRRIS, J. A. (1943). Habituatory response decrement in the intact organism. Psych. Bull. 40: 385-422.

Humphrey, G. (1933). The Nature of Learning and its Relation to the Living System. Harcourt, Brace and World, Inc., New York.

Jennings, H. S. (1906). Behavior of the Lower Organisms. Columbia University Press, New York.

Kinastowski, W. (1963a). Der Einfluss der mechanischen Reize auf die Kontraktilität von Spirostomum ambiguum Ehrbg. Acta Protozool. 1: 201-222.

Kinastowski, W. (1963b). Das Problem “des Lernes" bei Spirostomum ambiguum. Acta Protozool. 1: 223-236. 
McConnell, J. V. (1966). Comparative physiology: learning in invertebrates. Ann. Rev. Physiol. 28: 107-136.

ShARPLESS, S. K. (1964). Reorganization of function in the nervous system-use and disuse. Ann. Rev. Physiol. 26: 357-388.

Spencer, W. A., Thompson, R. F., and Nenlson, JR., D. R. (1966a). Response decrement of the flexion reflex in the acute spinal cat and transient restoration by strong stimuli. J. Neuróphysiol. 29: 221-239.

Spencer, W. A., Thompson, R. F., and D. R. Neilson, Jr. (1966b). Decrement of ventral root electrononus and intracellularly recorded post-synaptic potentials produced by iterated cutaneous afferent volleys. $\int$. Neurophysiol. 29: 253-274.

Thompson, R. F. and Spencer, W. A. (1966). Habituation: A model phenomenon for the study of neuronal substrates of behavior. Psychol. Rev. 73: 16-43.

Thorpe, W. H. (1956). Learning and Instinct in Animals. Harvard University Press, Cambridge.

Woodworth, R. S. (1936). Experimental psychology. Henry Holt, New York.

Wood, D. C. (1970). J. Neurobiol, in press.

The author would like to gratefully acknowledge the material support provided him by Dr. James Olds during the course of the studies and the critical discussion of the manuscript provided by the members of his doctoral committee; Drs. Olds, Edward L. Walker, James B. Ranck, Jr. and Stephen Glickman, at the University of Michigan. During the course of these studies the author was supported by a predoctoral fellowship provided by the N.I.H. 